\title{
Broadband THz response of a resonantly excited high-density exciton gas
}

\author{
Rupert Huber, Robert A. Kaindl, Benjamin A. Schmid, and Daniel S. Chemla \\ Department of Physics, University of California at Berkeley, and \\ Material Sciences Division, E.O. Lawrence Berkeley National Laboratory, Berkeley, California 94720, \\ USA
}

\begin{abstract}
The density-driven crossover of electron-hole pairs from insulating to conducting states is observed via the internal 1s-2p exciton resonance. Decreasing interparticle distance induces strong shifts and broadening, and ultimately the disappearance of the excitonic resonance.
\end{abstract}

In a dense electron-hole system, many-particle interactions govern its nature as a conductive plasma of unbound electron-hole $(e-h)$ pairs or an insulating gas of bound pairs (excitons). Recently, terahertz (THz) spectroscopy has provided direct experimental access to study the interplay between optically generated excitons and unbound carriers in semiconductor quantum wells [1]. Unlike near infrared and visible light, $\mathrm{THz}$ radiation resonantly probes internal transitions between bound states. Until now, these studies have been performed only for a dilute exciton gas. With increasing density, however, phase space filling, exchange interaction, and mutual screening lead to important modifications of the pair correlations. Ultimately, these many-body processes are expected to lead to the exciton-plasma Mott transition [2], a long standing topic of controversial debate.

Here, we employ time-resolved THz spectroscopy to probe the internal 1s-2p transition of resonantly photogenerated excitons at high densities. We directly trace changes of the far infrared resonances while the system undergoes a density-driven transition from an insulating to a conductive state. Our sample consists of a stack of ten high-quality, undoped 14-nm-wide GaAs wells separated by 10-nm-wide $\mathrm{Al}_{0.3} \mathrm{Ga}_{0.7}$ As barriers. All measurements are performed at low temperatures $(T=6 \mathrm{~K})$. The near-infrared absorption spectrum, shown in Fig. 1a, is dominated by the 1s heavy-hole (hh) exciton line at $1.54 \mathrm{eV}$. Spectrally shaped near-infrared pulses (red line, Fig. 1a) derived from a 250-kHz Ti:sapphire amplifier resonantly photoexcite heavy-hole excitons. At high pump fluences, the near-infrared absorption line
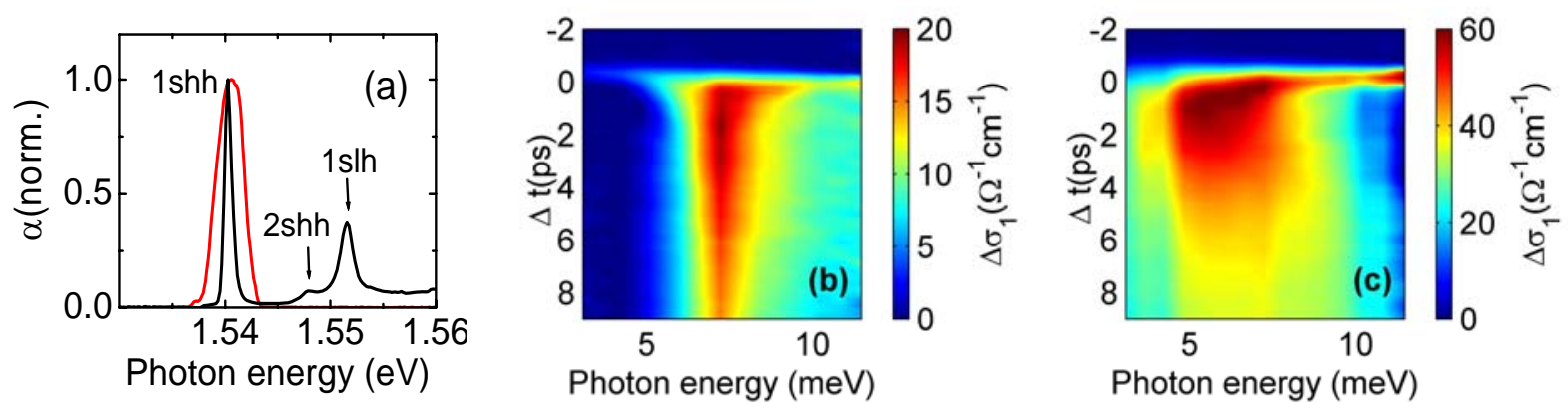

Fig. 1. (a) Near-infrared absorption spectrum of the GaAs multiple quantum wells at a temperature of $\mathrm{T}=6 \mathrm{~K}$ (black curve). The red curve represents the spectrum of the near-infrared pump pulses. (b) and (c) photoinduced conductivity change $\Delta \sigma_{1}$ measured by THz spectroscopy as function of frequency and delay time $\Delta t$ for and excitation density of $n_{0} \sim 10^{10} \mathrm{~cm}^{-2}$ (b) and $\mathrm{n} \sim 5 n_{0}$ (c). 

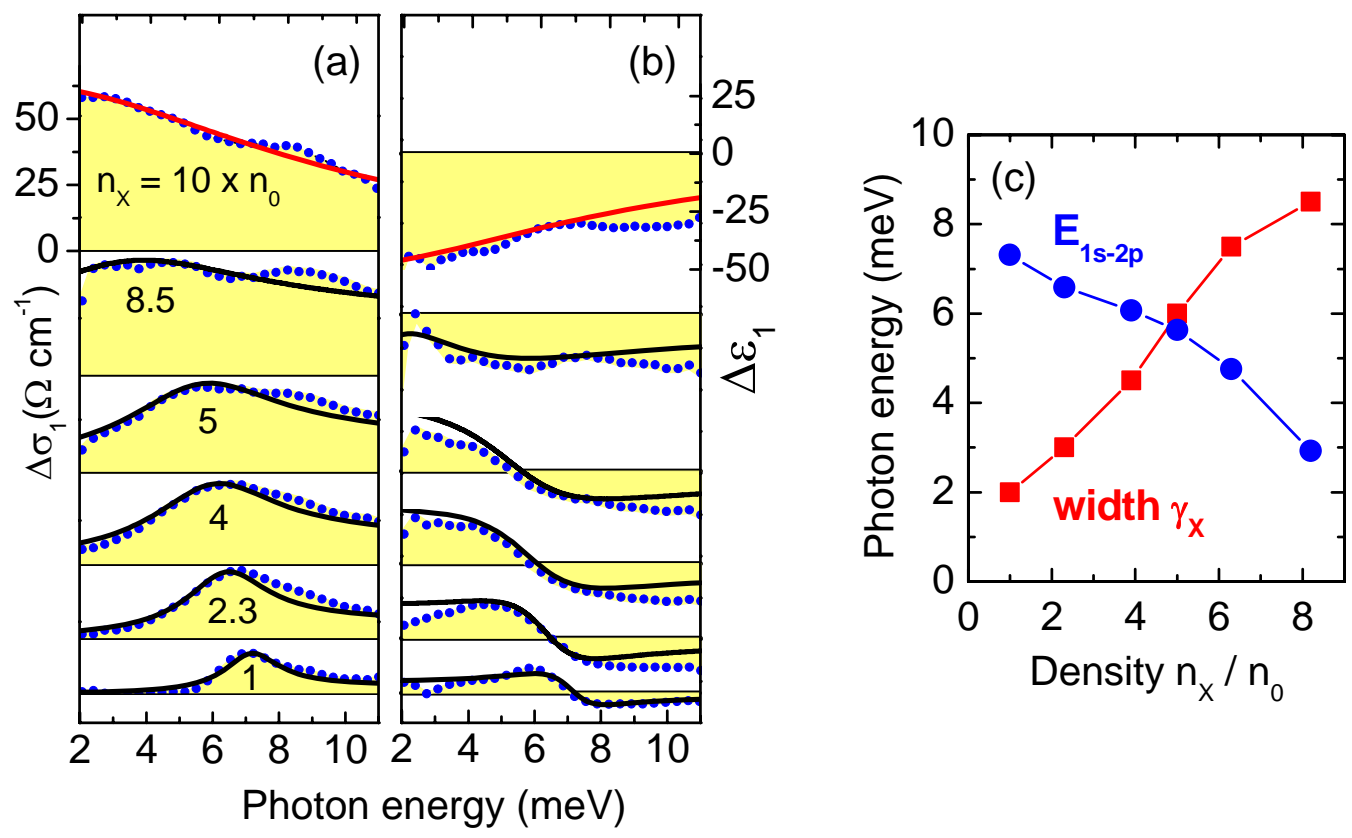

Fig. 2: (a) and (b) Induced THz response, $\Delta \sigma_{1}$ and $\Delta \varepsilon_{1}$, measured (blue dots) at delay $\Delta t=2.5 \mathrm{ps}$ for various excitation densities $n_{\mathrm{X}}$, with $\mathrm{n}_{0} \approx 1.5 \times 10^{10} \mathrm{~cm}^{-2}$. The curves are shifted vertically for clarity. Black lines: two-dimensional exciton model, red lines: Drude model. (c) Level spacing $E_{1 \mathrm{~s}-}$ ${ }_{2 p}$ and width $\gamma_{X}$ of the intra-excitonic $1 s-2 p$ transition as a function of the excitation density $n_{X}$ corresponding to the theory curves in Figs. 1b,c.

exhibits broadening, bleaching, and a slight blue shift. In order to photoinject high densities of excitons, we choose the spectral width of the pump ( $3.5 \mathrm{meV}$ FWHM) to be distinctly wider than the low-intensity absorption line (0.8 meV FWHM). Time-delayed THz probe pulses, generated by optical rectification and detected by electro-optic sampling in ZnTe, monitor the internal dynamics of the $e$ - $h$ pairs. Induced nearinfrared transmission changes can be measured concurrently. Comparing THz electric fields transmitted through the sample in equilibrium with pump-induced changes, we obtain the change of the complex response functions [1].

Ultrafast transient changes in the THz conductivity $\Delta \sigma_{1}$ after near infrared excitation are displayed in Figs. 1b,c, as a function of probe frequency $\omega$ and pump-probe delay time $\Delta t$. These curves, which are shown for two representative pump fluences, demonstrate a strong density dependence of the $\mathrm{THz}$ response. In the following, we concentrate on features that emerge within the time resolution of our experiment ( 2ps). Figures 2a,b show the response functions for various excitation densities $n_{\mathrm{X}}$ at fixed delay time $\Delta t=2.5 \mathrm{ps}$. At the lowest density of $n_{\mathrm{X}} \approx 1.5 \times 10^{10} \mathrm{~cm}^{-2}$, a narrow asymmetric peak in $\Delta \sigma_{1}(\omega)$ at $\hbar \omega=7 \mathrm{meV}$ demonstrates the existence of bound $e$-h pairs. The maximum arises from the $1 \mathrm{~s}-2 \mathrm{p}$ transition, while the high energy shoulder corresponds to transitions from 1s into higher-energy bound and continuum states. At low frequencies, the conductivity vanishes, giving rise to an insulating manybody system. The dispersive zero crossing of $\Delta \varepsilon_{1}$ at a photon energy of $7 \mathrm{meV}$ is characteristic of this well defined excitonic oscillator.

An increase of the excitation density results in two profound changes of the THz response: (i) both the maximum of $\Delta \sigma_{1}(\omega)$ and the zero crossing of $\Delta \varepsilon_{1}(\omega)$ shift to lower frequencies, (ii) the width of the observed features strongly increases. At the highest excitation density, the conductivity rises monotonically towards low frequencies and the zero crossing of the dielectric function is absent. This behavior is indicative of a conducting state without excitonic resonances. For a quantitative analysis, we fit the measured data in Figs. 2a,b with a model that takes into account the quasi-2D exciton wavefunctions (black lines, Figs. 2a,b). To this end, we vary the density $n_{\mathrm{X}}$, energy position $E_{1 \mathrm{~s}-2 \mathrm{p}}$, and 
width $\gamma_{\mathrm{X}}$ of the 1s-2p transition. The result of these fits shown in Fig. 2c underlines the striking densitydependent red-shift and broadening of the $1 \mathrm{~s}-2 \mathrm{p}$ transition. The exciton broadening gradually exceeds the level spacing. At the highest density, the response is well described by the Drude conductivity model (red line, Fig. 2a,b).

We emphasize two salient aspects of the THz response. First, the density-dependent broadening of the resonance is at least a factor of 3 larger than that of the near-infrared 1s exciton line [3]. This results from the particular sensitivity of the THz experiment to the 2p exciton state. Due to the larger spatial extent, the scattering rate of the p-like wave function is expected to be enhanced as compared to that of the $1 \mathrm{~s}$ exciton. Secondly, density-dependent experiments which probe the near-infrared exciton response are limited by the counteracting effects of bandgap renormalization and reduced exciton binding energy [4]. In contrast, the $\mathrm{THz}$ intra-excitonic transitions measured here are unaffected by bandgap shifts. In this way, our experiments provide a direct way to measure the effects of phase space filling and screening on exciton levels in a high density gas of $e$ - $h$ pairs.

Acknowledgements This work was supported by the Director, Office of Science, Office of Basic Energy Sciences, Division of Materials Sciences, of the US Department of Energy under Contract No. DE-AC0205CH11231, and through a fellowship of the Alexander von Humboldt foundation.

\section{References}

[1] R. A. Kaindl, M. A. Carnahan, D. Hägele, R. Lövenich, and D. S. Chemla, Nature 423, 734 (2003) and references therein.

[2] N. F. Mott, Philos. Mag. 6, 287 (1961).

[3] see e.g. A. Honold, L. Schultheis, J. Kuhl, and C. W. Tu, Phys. Rev. B 40, 6442 (1989).

[4] N. Peyghambarian, H. M. Gibbs, J. L. Jewell, A. Antonetti, A. Migus, D. Hulin, and A. Mysyrowicz, Phys. Rev. Lett. 53, 2433 (1984); Choi, K.-C. Je, S.-Y. Yim, and S.-H. Park, Phys. Rev. B 70, 085309 (2004).

\section{DISCLAIMER}

This document was prepared as an account of work sponsored by the United States Government. While this document is believed to contain correct information, neither the United States Government nor any agency thereof, nor The Regents of the University of California, nor any of their employees, makes any warranty, express or implied, or assumes any legal responsibility for the accuracy, completeness, or usefulness of any information, apparatus, product, or process disclosed, or represents that its use would not infringe privately owned rights. Reference herein to any specific commercial product, process, or service by its trade name, trademark, manufacturer, or otherwise, does not necessarily constitute or imply its endorsement, recommendation, or favoring by the United States Government or any agency thereof, or The Regents of the University of California. The views and opinions of authors expressed herein do not necessarily state or reflect those of the United States Government or any agency thereof or The Regents of the University of California. 